\title{
Is oral lichen planus a risk factor for peri- implant diseases? A systematic review and meta-analysis
}

\author{
Xiaoqin Xiong ${ }^{\dagger}$, Tiantian $\mathrm{Xu}^{\dagger}$, Xinhong Wang, Wenguang Qin, Ting Yu ${ }^{*}$ [D and Gang Luo
}

\begin{abstract}
Background: To evaluate whether oral lichen planus (OLP) is a risk factor for peri-implant diseases (PIDs) with a systematic review and meta-analysis.

Methods: Five electronic databases including Medline, Embase, Web of Science, the Cochrane Library and Scopus were searched. The included studies are observational human studies written in English. The population of interest included those with/without OLP who received dental implant treatment. The follow-up time after implantation was from 1 month to 20 years. The quality of the included articles regarding risk of bias and methodology were assessed with the Newcastle-Ottawa Scale or the Agency for Healthcare Research and Quality. The data involving exposure (OLP), primary outcomes (implants having PIDs) and secondary outcomes (probing depth/PD, bleeding on probing/BOP and bone loss/BL) and potential confounders were extracted. Heterogeneity was assessed by $\mathrm{I}^{2}$ test. Dichotomous data are expressed as the risk ratio (RR) and 95\% confidence interval (Cl) which were calculated with a fixed effect model.
\end{abstract}

Results: Of the 66 articles, two studies were enrolled and evaluated as high quality, which totally contained 68 participants receiving 222 (OLP vs. non-OLP, 112 vs. 110) implants with 12 to 120-month follow-up time. Proportions of implants with PIDs between OLP and non-OLP groups were as follows: 19.6\% (22/112) vs. 22.7\% (25/ 110) for PIM and $17.0 \%$ (19/112) vs. 10.9\% (12/110) for PI. The meta-analysis revealed no recognizable difference in number of implants with PIDs (PI: RR $=1.49,95 \% \mathrm{Cl} 0.77-2.90, P=0.24$; PIM:RR $=0.88,95 \% \mathrm{Cl} 0.53-1.46, P=0.61$; PIDs: $\mathrm{RR}=1.08,95 \% \mathrm{Cl} 0.75-1.55, P=0.68)$ or $\mathrm{BOP}(\mathrm{RR}=0.90,95 \% \mathrm{Cl}: 0.70-1.15, P=0.40)$ between OLP and non-OLP groups.

Conclusions: Available articles regarding the effects of OLP on PIDs remains very limited. Existing evidence does not support OLP as a suspected risk factor for PIDs. Large-scale prospective trials are required to validate the findings.

Keywords: Dental implants, Peri-implant diseases, Oral lichen planus, Systematic review, Meta-analysis

\footnotetext{
*Correspondence: dent_yu@163.com; gangluo@139.com

${ }^{+}$Xiaoqin Xiong and Tiantian Xu contributed equally to this work.

Department of Periodontology and Oral Medicine, Key Laboratory of Oral

Medicine, Guangzhou Institute of Oral Disease, Stomatology Hospital of

Guangzhou Medical University, NO.195 Dongfeng West Road, Guangzhou

510140, China
}

\section{$\triangle B M C$}

(c) The Author(s). 2020 Open Access This article is licensed under a Creative Commons Attribution 4.0 International License, which permits use, sharing, adaptation, distribution and reproduction in any medium or format, as long as you give appropriate credit to the original author(s) and the source, provide a link to the Creative Commons licence, and indicate if changes were made. The images or other third party material in this article are included in the article's Creative Commons licence, unless indicated otherwise in a credit line to the material. If material is not included in the article's Creative Commons licence and your intended use is not permitted by statutory regulation or exceeds the permitted use, you will need to obtain permission directly from the copyright holder. To view a copy of this licence, visit http://creativecommons.org/licenses/by/4.0/. The Creative Commons Public Domain Dedication waiver (http://creativecommons.org/publicdomain/zero/1.0/) applies to the data made available in this article, unless otherwise stated in a credit line to the data. 


\section{Background}

Dental implant treatment is a well-established method for restoring lost teeth. Despite a high rate of survival (> $94 \%)$ for more than 5 years [1, 2], a very high proportion (8-44\%) of dental implants develop peri-implant diseases (PIDs), including peri-implant mucositis (PIM) and peri-implantitis (PI) [3], which can lead to implant failure or loss. PIDs are pathological inflammatory conditions, of which PIM involves only peri-implant mucosal inflammation, while PI causes both peri-implant mucosa and alveolar bone damage [4], accompanied by bleeding on probing, suppuration, increased probing depth, and progressive marginal bone loss [5]. PID-associated risk factors include poor oral hygiene [6], smoking [7], a history of periodontitis $[4,8]$ and systemic diseases, such as diabetes [9].

Oral lichen planus (OLP), a chronic systemic autoimmune disease, affects one to $2 \%$ of the general population, especially middle-aged and elderly females [10]. Typically, OLP manifests as plaque-like or white reticular lesions, an erythematous area or ulceration $[11,12]$, and it can affect the oral mucosa, tongue or gingiva [13]. Recently, OLP has been questioned to be a potential risk indicator for PIDs. OLP patients have been found to have poor oral hygiene [14] and quality of life [15]. Furthermore, some studies have shown a very high implant failure rate (42/55) for OLP patients receiving implant placement during the acute stages [16], some researchers have also demonstrated that OLP patients feel more stress and have a weaker psychological profile [17]. These concerns have previously made some dentists consider OLP a contraindication to implant treatment. However, some studies, in which 66 short implants were implanted in 23 OLP patients with a survival rate of $98.5 \%$, did not observe any difference in the success rate between OLP patients and normal controls [18, 19]. Notably, most of the studies regarding implantation in OLP populations were observational studies (including case reports, retrospective studies, etc.) with small sample sizes. Hence, whether dental implantation in OLP patients is safe remains inconclusive. The present systematic review aimed to analyze the existing articles to determine whether OLP is a risk factor for PIDs.

\section{Methods}

\section{Focused question}

Compared with non-OLP populations, are there changes in the rates of probing depth, bone loss or bleeding on probing around implants in OLP patients? Are there different rates of PIM and PI prevalence in OLP patients compared with non-OLP populations?

This manuscript has been prepared based on the Preferred Reporting Items for Systematic Review and Meta- analyses (PRISMA) guidelines for reporting systematic reviews.

\section{Population and exposure}

The population of interest was patients with and without OLP who received dental implant treatment. The studies included participants aged $\geq 18$ years with no periodontitis or stable periodontitis after treatment. Their OLP status was diagnosed before implant treatment. It was preferred that OLP patients had a diagnosis of the OLP subtype or concomitant symptoms, such as desquamative gingivitis which could be considered separate exposure factors. Detailed records of treatments, including medications, were included for all OLP patients. The primary outcomes were PIDs, and the secondary outcomes were probing depth, bleeding on probing, and bone loss. Implant failure was also considered if its infective origin was clearly stated.

\section{Disease determinants, risk factors, and etiologic agents}

The potential association of OLP with PID determinants, including sex, age, educational background, socioeconomic position, genetics, lifestyle, nutrition, health behaviors, and microbiological factors, was recorded when available. Whenever possible, the quality of the determinant/exposure measurements was assessed.

There are some general risk indicators/factors for PI and PIM, including a history of periodontitis [20], systemic diseases, genetic traits, and smoking. Local risk indicators/factors for PI and PIM include oral hygiene/poor plaque control, poor compliance with supportive implant therapy, presence of excess cement, implant materials and surface characteristics [21], design of implant-supported prostheses and dimensions of keratinized mucosa $[4,8]$.

\section{Study follow-up duration}

All studies with a mean duration or follow-up interval of at least 1 month were included because the earliest time for PID diagnosis was approximately 1 month after loading restorations $[4,22]$. If the follow-up time was reported as a range without means or medians, the minimum should be no less than 1 month.

\section{Types of studies}

With the goal of identifying studies for inclusion, certain methods were designed based on acceptability criteria in this study. Eligible studies, including observational or longitudinal studies or prospective research, were those with a follow-up time $\geq 1$ month, with primary outcomes of PIDs and implants loss or failure. The secondary outcomes were probing depth, bleeding on probing and bone loss. 


\section{Inclusion criteria}

1) Observational human studies, such as case-control, cross-sectional, retrospective or prospective cohort studies;

2) Studies in which OLP and PIDs were the main exposure factor and outcome, respectively;

3) Both OLP and PIDs should be definitely diagnosed in studies, the diagnosis of OLP was made according to clinical and pathological evidence, the diagnosis of PIDs based on clinical settings, soft tissue inflammation was detected by detecting BOP, BL should be confirmed by PD and radiographic examinations.

4) Studies with a follow-up time of $\geq 1$ month after implant placement; and

5) Studies published in the English language.

\section{Exclusion criteria}

1) Studies lacking control groups (i.e., a non-OLP group);

2) Narrative reviews, comments, letters, case reports or series, and conference abstracts; and

3) Duplicates were removed, and the latest or most complete literature was retained.

\section{Search strategy}

The search was performed in June 2019. All available articles published online before this time in five electronic databases, including Medline, Embase, Web of Science, the Cochrane Library and Scopus, were retrieved.
Manual searching in dental journals, especially those focusing on implantology, was also performed. The search strategy was established with adequate discussion among all the coauthors (Table 1).

\section{Study selection}

Two researchers (XX and XT) screened the articles via abstract and full-text review independently and in duplicate. Afterward, articles that met the research topic were enrolled for full-text screening. If an article could not be excluded/included based on its title and abstract, it will be also subject to full-text review. Subsequently, the articles were independently assessed in duplicate by the reviewers. Any disparity about research eligibility was settled by discussion among the two reviewers, and if no consensus could be reached, the decision was resolved through arbitration by a third reviewer (YT). All articles that did not fulfill the eligibility criteria were excluded.

For studies with missing data or those for which a clear decision on study eligibility could not be made after assessment of the full text, the corresponding authors were e-mailed to ask for the information needed for a final decision. Messages via social media or academic websites were also sent to the corresponding authors in the absence of response by e-mail.

\section{Data extraction and management}

A detailed form designed specifically for data extraction was used, and all the relevant data from the collected articles were independently extracted by two independently. Any disagreements were settled through evaluation by a third examiner (YT) or group consensus.

Table 1 Electronic databases used and search strategies

\begin{tabular}{|c|c|c|c|}
\hline & Database/journal & Search strategy & $\begin{array}{l}\text { Items } \\
\text { found }\end{array}$ \\
\hline \multirow{5}{*}{$\begin{array}{l}\text { Electronic } \\
\text { searching }\end{array}$} & Medline & \#1 [All Fields] AND \#2 [All Fields] AND \#3[All Fields] & 24 \\
\hline & Embase & \#1:ti, ab, kw AND \#2:ti, ab, kw AND \#3:ti, ab, kw & 10 \\
\hline & Web of Science & $\mathrm{TS}=\# 1 \mathrm{AND} T \mathrm{TS}=\# 2 \mathrm{AND} \mathrm{TS}=\# 3$ & 11 \\
\hline & Cochrane Library & $\# 1: t i, a b, k w$ AND \#2:ti, ab, kw AND \#3:ti, ab, kw & 1 \\
\hline & Scopus & $\begin{array}{l}\text { TITLE-ABS-KEY (\#1) AND TITLE-ABS-KEY (\#2) AND TITLE-ABS-KEY } \\
\text { (\#3) }\end{array}$ & 11 \\
\hline \multirow[t]{7}{*}{ Manual searching } & Journal of Periodontology & $\# 1$ AND \#2 AND \#3 & 1 \\
\hline & $\begin{array}{l}\text { Clinical Implant Dentistry and Related } \\
\text { Research }\end{array}$ & $\# 1$ AND \#2 AND \#3 & 3 \\
\hline & Clinical Oral Implants Research & \#1 AND \#2 AND \#3 & 1 \\
\hline & Journal of Clinical Periodontology & $\# 1$ AND \#2 AND \#3 & 1 \\
\hline & British Journal of Oral \& Maxillofacial Surgery & $\# 1$ AND \#2 AND \#3 & 1 \\
\hline & Clinical Oral Investigations & $\# 1$ AND \#2 AND \#3 & 1 \\
\hline & Journal of Dentistry & $\# 1$ AND \#2 AND \#3 & 1 \\
\hline
\end{tabular}

\#1: "dental implant" OR "implant"; \#2 "peri-implantitis" OR "peri-implant disease" OR "peri-implant mucositis" OR "Ioss" OR "failure" OR "success rate" OR "survival rate"; \#3 OLP OR "oral planus lichen" 
Table 2 General description of the included studies

\begin{tabular}{|c|c|c|}
\hline Study & Gonzalo Hernandez (2012) & Pia López-Jornet (2014) \\
\hline Age (year) & Median (range) 53.7 (38-73)/52.2 (35-70) & Median (range) 64.5 (44-76)/42 (29-79) \\
\hline OLPG/ CG & $18 / 18$ & $16 / 16$ \\
\hline Male/Female & $10 / 26$ & $14 / 18$ \\
\hline No. of implants & $56 / 60$ & $56 / 50$ \\
\hline $\begin{array}{l}\text { Definition of } \\
\text { OLP }\end{array}$ & $\begin{array}{l}\text { Clinical and histopathological criteria of OLP according to the } \\
\text { modified WHO diagnostic criteria of OLP }\end{array}$ & $\begin{array}{l}\text { OLP was diagnosed based on a thorough clinical } \\
\text { examination and histopathology of the lesions }\end{array}$ \\
\hline $\begin{array}{l}\text { Definition of PIM } \\
\text { and PI }\end{array}$ & $\begin{array}{l}\text { The presence of PIM (BOP, PD } \geq 4 \mathrm{~mm} \text { and } \mathrm{no} \mathrm{BL} \text { ) and } \mathrm{PI} \text { (BOP or } \\
\text { pus, } \mathrm{BL} \geq 3 \text { threads at the final examination) }\end{array}$ & $\begin{array}{l}\text { Diagnosis of PIDs based on clinical indicators (e.g. CAL, PD, } \\
\text { BL) }\end{array}$ \\
\hline $\begin{array}{l}\text { Follow-up time } \\
\text { (months) }\end{array}$ & Median (IR): 56.5 (22)/52.5 (22.7) & Median (range): 42 (12-120)/48 (24-48) \\
\hline PD (mm): & PD (mm): $\mathrm{n}:<4$ mm 23/18; $\geq 4$ mm 33/42 & PD (mm): median (range): 3.00 (1.12-4.90)/3.00 (2-5) \\
\hline BOP & (sites): 105/114; (implants): 36/44; patients: 13/16 & (No. implants): 12/11 \\
\hline$B L$ & $\mathrm{BL}(\mathrm{mm}): \leq 1.718 / 22 ; 1.8-2.424 / 24 ; 2.5-38 / 9 ; 3.1-3.64 / 3 ; \geq 3.7: 2 / 2$ & (No. of implants): 10/8; \\
\hline $\begin{array}{l}\text { PIM (No. of } \\
\text { implants) }\end{array}$ & $12 / 16$ & 10/9 \\
\hline $\begin{array}{l}\text { PI (No. of } \\
\text { implants) }\end{array}$ & $5 / 4$ & $14 / 8$ \\
\hline $\begin{array}{l}\text { Confounders } \\
\text { controlled for }\end{array}$ & Smoking & $\begin{array}{l}\text { Age, sex, smoking, alcohol consumption, frequency of tooth } \\
\text { brushing }\end{array}$ \\
\hline Key findings & $\begin{array}{l}\text { Lichen planus was not a prominent local factor in the } \\
\text { genesis of implant failure. }\end{array}$ & $\begin{array}{l}\text { Implants did not influence manifestations of OLP. OLP was } \\
\text { not a risk factor for peri-implantitis. }\end{array}$ \\
\hline Odds Ratio & - & $1.32(\mathrm{PI})$ \\
\hline $\begin{array}{l}95 \% \text { Confidence } \\
\text { Interval }\end{array}$ & - & $0.81-2.14(\mathrm{PI})$ \\
\hline$p$ Value & $.254(\mathrm{PI}) / .985$ (PIM) & $.257(\mathrm{PI})$ \\
\hline
\end{tabular}

$M / F$ male/female, $O L P G$ oral lichen planus group, $C G$ control group, $B L$ bone loss, $B O P$ bleeding on probing, $C A L$ clinical attachment level, $P D$ probing depth, $P I$ peri-implantitis, PIM peri-implant mucositis, $S$ systemic diseases

The following research information was extracted (Table 2):

1) Type of research;

2) Sample framework (e.g., community, university);

3) Sample size, sex and age of participants;

4) Diagnostic criteria for PI, PIM and OLP;

5) Follow-up time; and

6) Confounding factors relative to exposure factors.

\section{Outcomes}

1) Probing depth change;

2) Bone loss level change;

3) Bleeding on probing level change;

4) Number of implants with/without PI and PIM; and

5) Number of implants loss or failure.

\section{Quality assessment}

The Newcastle Ottawa Scale (NOS) [23] and the Agency for Healthcare Research and Quality (AHRQ) [24] standards were used to assess the quality of cohort and cross-sectional studies respectively. The cohort study degree of bias was categorized with a star-based quality assessment system that considers three categories as follows: the selection of the study groups (four stars), the comparability of the groups (two stars), and the ascertainment of either the exposure or outcome of interest (three stars). An 11-item checklist recommended by the AHRQ was used to assess the methodological quality of the cross-sectional studies. Article quality was assessed as follows: low quality (0 to 3 ); moderate quality (4 to 7 ); and high quality (8 to 11 ).

\section{Data synthesis}

Statistical heterogeneity in risk in patients suffering from PIDs in the collected studies was evaluated using both risk ratios (RRs) and $\mathrm{I}^{2}$ measures. The use of $\mathrm{I}^{2}$ values was based on the Cochrane Handbook: $0<\mathrm{I}^{2}<40 \%$ represents a lack of heterogeneity; $30 \%<\mathrm{I}^{2}<60 \%$ represents moderate heterogeneity; $50 \%<\mathrm{I}^{2}<90 \%$ signals substantial heterogeneity; and $75 \%<\mathrm{I}^{2}<100 \%$ shows considerable heterogeneity.

When suitable, meta-analysis was used to assess risk in patients suffering from PIDs based on implant and patient characteristics. A random effects approach was 
used if there was mild to moderate statistical heterogeneity, otherwise, a fixed-effect approach was chosen. Considering that only observational studies were included in the present systematic review, the primary outcome variable was the RRs of PI and PIM. The RRs were calculated with 95\% confidence intervals (CIs) (Review Manager, v5.2, The Nordic Cochrane Center, The Cochrane Collaboration, Copenhagen, Denmark) to quantify the association between OLP and the risk of PI and PIM. Forest plots were generated showing the RRs and 95\% CIs of the involved studies. If there were more than 10 studies included in the meta-analysis, publication bias was evaluated qualitatively by a funnel plot [25].

\section{Results}

\section{Search}

A total of 66 studies were identified after electronic and manual searches (Fig. 1). After removing duplicates, 26 articles were retained. Then, 7 articles were excluded after evaluating the titles and abstracts, and the remaining 19 articles were selected for full-text evaluation. Of the 19 articles, 13 articles involved both OLP and implant treatment. Then, 11 articles were excluded for various reasons, such as being case reports, reviews, etc., and the remaining 2 articles were retained for final review. According to the Inclusion and exclusion criteria, there was no studies available on implant loss or failure as the primary outcome. The screening outcomes were similar regardless of whether the article was published in English [26].

\section{Study characteristics}

\section{Location and sample characteristics}

The two included studies included one prospective study and one cross-sectional study [15] (Table 2). Both studies were conducted in the same country (Spain) but by different institutions. The two studies contained 34 OLP patients and 34 non-OLP controls aged between 29 and 79 years. 68 participants received 224 implants (OLP vs. non-OLP, 112 vs. 110) with a follow-up time of 12 to 120 months.

At the implant level, 19.6\% (22/112) and 22.7\% (25/ 110) developed PIM in the OLP and non-OLP groups,

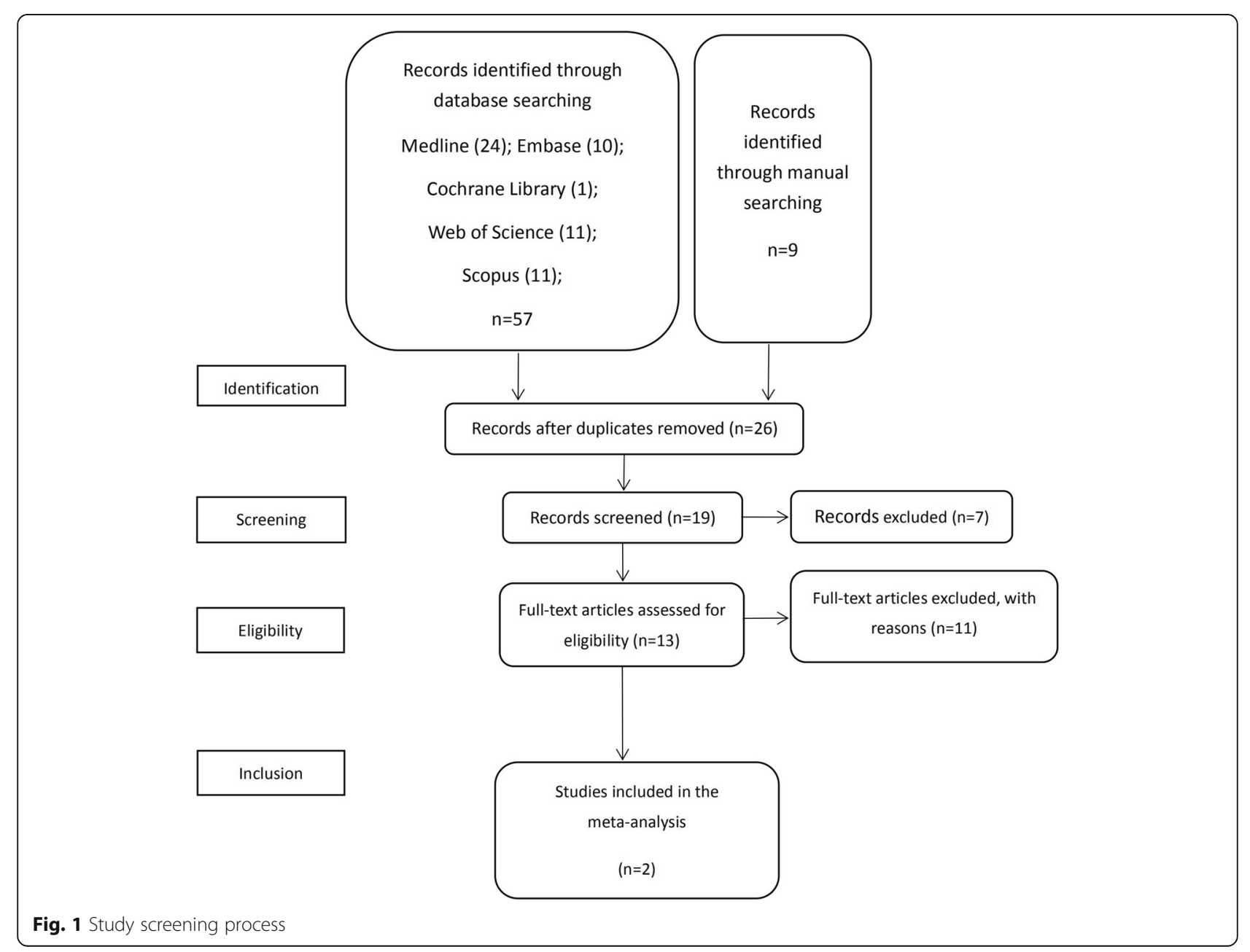


Table 3 Quality assessment of the prospective study

\begin{tabular}{llllll}
\hline Study & Selection (max 4 asterisks) & Comparability (max 2 asterisks) & Exposure (max 3 asterisks) & Score & Quality \\
\hline Gonzalo Hernandez (2012) & $*^{* *}$ & ${ }^{* *}$ & ${ }^{* *}$ & 7 & Low risk \\
\hline
\end{tabular}

while 17.0\% (19/112) and 10.9\% (12/110) developed PI in the two groups, respectively. Additionally, bleeding on probing was present at the sites of $42.6 \%(48 / 112)$ and $50.0 \%(55 / 110)$ of implants in the two groups, respectively.

The ratios of PIDs in two groups could not be calculated at the individual level due to the missing data. The outcomes regarding probing depth and bone loss could not be combined because they were reported in different forms, and only one study's authors provided the original data for these parameters by online contact.

\section{Risk of bias and methodological quality}

Quality evaluation according to the NOS showed that the prospective study was rated as having 7 stars and was classified as high quality (Table 3 ). Based on the AHRQ criterion, the final score of the cross-sectional study was 8 , and it was classified as having high quality (Table 4).

\section{Risk of PIDs in the OLP versus non-OLP groups}

Due to the small sample size and good homogeneity $\left(X^{2}=0.04, P=0.84 ; I^{2}=0\right)$ of the included articles, the fixed-effect model was employed to calculate the pooled

Table 4 Quality assessment of the cross-sectional study

\begin{tabular}{ll}
\hline Items & Yes No Unclear \\
\hline 1) Define the information (survey, record review) & 1 \\
2) List inclusion and exclusion criteria for exposed & 1 \\
and unexposed subjects (cases and controls) or refer \\
to previous publications \\
3) Indicate time period used for identifying patients & 1 \\
4) Indicate whether or not subjects were consecutive & 1 \\
if population-based \\
5) Indicate if evaluators of study subjects were \\
blinded to other aspects of the status of the \\
participants \\
6) Describe any assessments undertaken for quality & 1 \\
assurance purposes (e.g., test/retest of primary \\
outcome measurements) \\
7) Explain any patient exclusions from analysis \\
8) Describe how confounding was assessed and/or \\
controlled \\
9) If applicable, explain how missing data were \\
handled in the analysis \\
10) Summarize patient response rates and \\
completeness of data collection \\
11) Clarity what follow-up, if any, was expected and \\
the percentage of patients for which incomplete \\
data or follow-up was obtained \\
\hline
\end{tabular}

$R R$ in this meta-analysis. The model showed no significant difference in the number of implants suffering from PI $(R R=1.49,95 \% C I: 0.77-2.90, P=0.24$, Fig. $2 \mathrm{a})$ or PIM $(R R=0.88,95 \% C I$ : $0.53-1.46, P=0.61$, Fig. $2 \mathrm{~b})$ between the OLP and non-OLP groups at the implant level. There was a similar result when combining PI and PIM into PID $(R R=1.08,95 \% C I: 0.75-1.55, P=0.68$, Fig. 2c). This was also the case for the presence of bleeding on probing between the two groups $(R R=0.90,95 \%$ CI: 0.70-1.15, $P=0.40$, Fig. 2d).

\section{Discussion}

\section{Key findings}

The present systematic review assessed the current existing evidence on the relationship between OLP and PIDs for the first time and attempted to determine whether OLP is a potential risk factor for PIDs. Of 139 studies, two, which contained 68 participants receiving 222 (OLP vs. non-OLP, 112 vs. 110) implants, with a 12 - to 120 month follow-up time, were included and evaluated as having high quality. The proportions of implants with PIDs between the OLP and non-OLP groups were as follows: $19.6 \%(22 / 112)$ vs. $22.7 \%$ (25/110) for PIM and $17.0 \%(19 / 112)$ vs. $10.9 \%(12 / 110)$ for PI. Existing evidence seems to suggest that OLP is not a suspected risk factor for PIDs during the 10-year follow-up period.

\section{Overall completeness and applicability of the evidence}

The diagnostic criteria for OLP were clearly stated in the two enrolled studies, while those for PIDs were absent in the cross-sectional study. Moreover, in the two enrolled studies, PIDs were evaluated at only the implant level and not at the individual level, while OLP was diagnosed at the individual level. Hence, the evidence was insufficient to determine whether OLP is a risk factor for PIDs at the individual level.

Concerning secondary outcomes of PIDs, both studies had complete bleeding on probing data. After data combination, OLP was found to not be a risk factor for bleeding on probing at the implant level. For the secondary outcomes for PIDs, i.e., probing depth and bone loss, the data were complete but reported in different forms in the two studies. The prospective study set cutoff values for probing depth and bone loss to determine the percentages of involved implants, while the crosssectional study directly presented the medians and ranges of the two parameters. Despite repeated contact with the authors, we obtained only the raw data of the prospective study. Hence, an attempt to combine the 


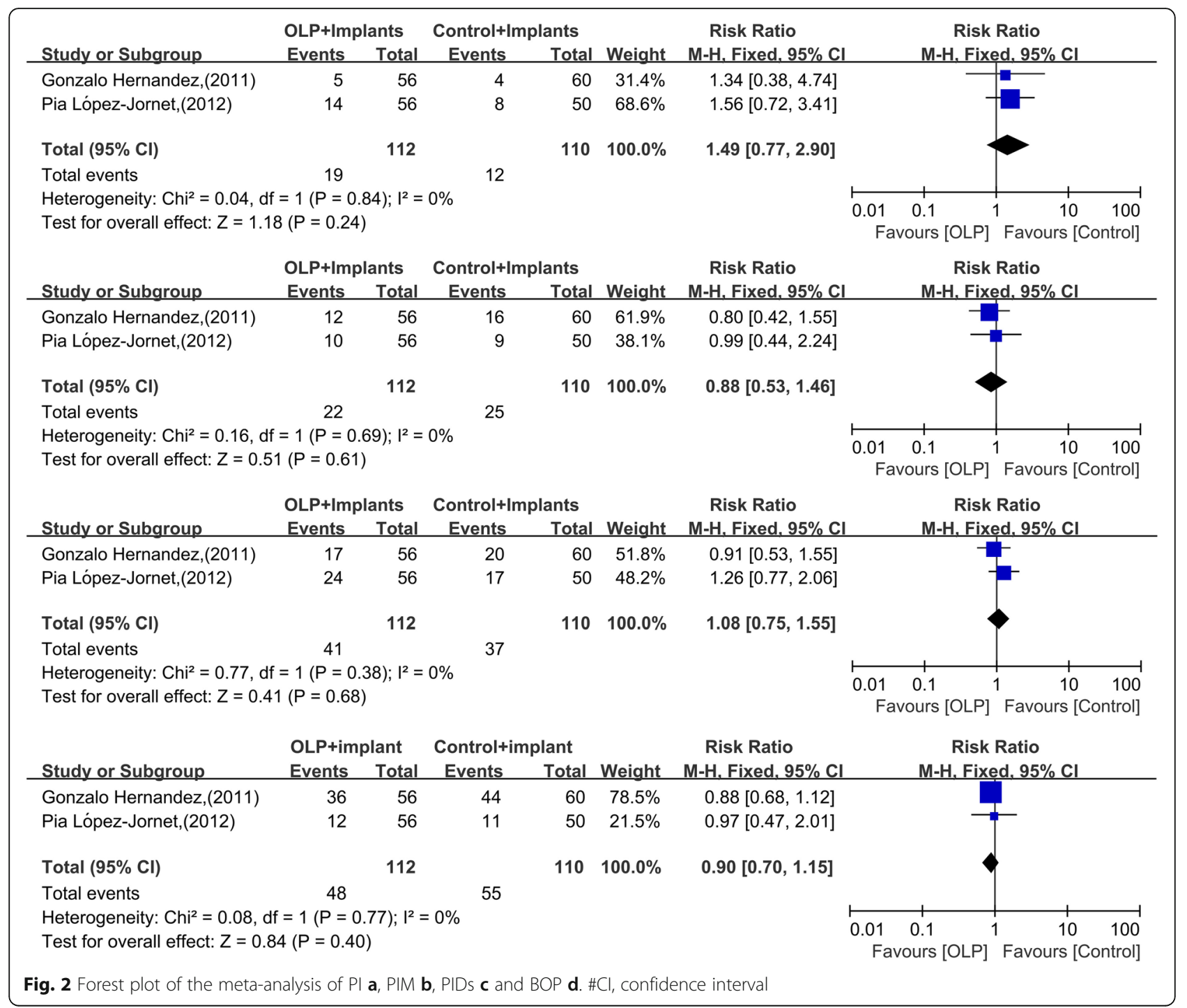

data of the two studies in terms of probing depth or bone loss could not be achieved. Ultimately, both studies found no significant difference in probing depth or bone loss between the OLP and non-OLP groups.

When the participant was used as the unit of analysis, the intragroup analysis of the OLP group failed to demonstrate a statistically significant relationship between the existence of desquamative gingivitis and PIM or desquamative gingivitis and PI, but 20 out of 25 implant patients with PIM were among those with desquamative gingivitis in the OLP group; hence, PIM with DG in the OLP group occurred more frequently than nondesquamative gingivitis in the OLP group in this subgroup of implant patients in the prospective study [27]. Since the cross-sectional study presented insufficient information, although the corresponding authors were contacted in various ways and only the author of the prospective study provided the original data of the parameters of interest, no subgroup analysis was performed. Therefore, subgroup combinations in the metaanalysis were not possible. We considered implant failure as an outcome but get no eligible article. Five articles were excluded in the screening step due to case reporting, no control group, etc. Despite no parallel studies comparing implant failure between OLP patients and non-OLP controls, OLP patients have shown a similar implant failure rate $(2.7 \%, 14 / 523)$ to populations without OLP $(\sim 2 \%)[28]$.

Overall quality, strength, and consistency of the evidence The present systematic review included only two observational studies that met the inclusion criteria mainly because related research on implantation in OLP populations is scarce. However, the durations of the two enrolled studies were very similar (2003 to 2009 vs. 2005 to 2010), and the study populations were from the same 
country, which may be beneficial for data synthesis. Nevertheless, the results might be more applicable to specific ethnicities or countries and may not be easily generalized for people globally. The average age of the OLP patients in the two studies was quite different (64.5 vs. 53.7). This distinction may introduce uncertainty regarding the applicable age after pooling the data. Additionally, the mean follow-up time of the two studies was similar but did not exceed 5 years; therefore, it is unclear whether OLP is a risk factor for PIDs over an observation period longer than 5 years. The two studies contained detailed records of important confounders, such as smoking, but only the prospective study controlled for confounders. The response rate was not reported in either study; thus, the number of lost samples was unknown. In the prospective study, two implants in the non-OLP group were lost for unknown reasons, and the study did not state whether it was a PI-associated consequence. Even if that was the case, there was no difference in the results of the PI $(R R=1.31,95 \% C I: 0.70$ $2.45, P=0.40)$ analysis between the two cases.

Case definitions for PIDs vary considerably in previous studies [8], and PID data were especially challenging to interpret. Only the prospective study, which reported clear definitions of PIM and PI, was evaluated according to the defined criteria. The crosssectional study did not specify the diagnostic criteria of PIDs in detail, while the clinical indicators (e.g. $\mathrm{BOP}, \mathrm{PD}, \mathrm{BL}$ ) were described in detail with table. The two studies had different definitions of diseases, and there may have been some bias in the data. The diagnostic criteria for PI in the study were not uniform, and limited studies were included in the metaanalysis, so no meta-analysis could be conducted to prove the effect of the differences on the metaanalysis outcomes. Clinical indicators such as bleeding on probing and bone loss are relatively easy to standardize, although no effect of OLP on these indicators has been observed. Implant position has been considered as a prognostic factor for implant success [29]. However, we could not perform subgroup analysis with implant position as a grouping variable due to lack of raw data. Anyway, implants from the two studies showed equal distributions in jaws (maxilla/ mandible: $52 / 60$ vs. $63 / 49, X^{2}=2.162, P=0.141$ ) and tooth sites $\left(20 / 92\right.$ vs. $\left.23 / 89, X^{2}=0.259, P=0.611\right)$ between OLP and non-OLP groups, suggesting implant position might be not a source of heterogeneity to affect the conclusions.

The results of the statistical analysis show that the homogeneity between the results was good. However, the limited number of enrolled studies made it unfeasible to draw a funnel plot to assess the variation in the studies. Although the sample size was small, the strength of the study evidence was high as analyzed by qualitycontrol assessment, and the overall estimate of the meta-analysis represents the best available evidence.

\section{Implications for practice and policy}

Although there was no clear association between OLP and the occurrence of PIDs, implant-related issues in OLP patients cannot be easily ignored, and several issues are worth noting. First, in a clinical study [14], the gingival index of OLP group was calculated, and the mean value of periodontal index and rate of bleeding on probing were higher than those in control group, revealing that the periodontal condition in the OLP group was poor compared with that in the control group. Poor oral hygiene is a well-known risk factor for PIDs, therefore, OLP may have a certain impact on PIDs. Second, some scholars believed that the capacity of epithelium to adhere to the titanium surface of the implants in OLP patients may be affected because the adhesion of epithelial cells decreases, affecting the epithelial barrier around the implant surface [30]. Third, desquamative gingivitis is a special type of OLP that causes gingival damage. An increased frequency of PIM was reported in cases of desquamative gingivitis compared with non-desquamative gingivitis OLP patients [27]. Another study demonstrated that the presence of desquamative gingivitis was associated with relatively deep periodontal pockets [31] . The study inferred that desquamative gingivitis lesions might circuitously enhance the long-term risk for periodontal diseases via plaque accumulation by impeding proper oral hygiene around natural teeth and implants. It is important to clarify the constructive associations between desquamative gingivitis and PIDs in the future. Moreover, OLP is a T cell-mediated chronic inflammatory autoimmune disease [32]. PIDs in humans cause leukocytic infiltration through barrier epithelial migration and an increase in the proportion of $\mathrm{T}$ and $\mathrm{B}$ cells [33]. In this context, these two conditions might aggravate each other via inflammatory pathways. Additionally, dysbiosis might be a potential link between OLP and PIDs, given that both conditions have been related to microbial alterations [34, 35] . Finally, systemic corticosteroid treatment in OLP patients was frequently associated with decreased bone mineral density, especially during the first 6 months of corticosteroid therapy [36]. The osteoporotic effects of corticosteroids might cause more alveolar bone loss around dental implants during infection [37].

In this systematic review, the available evidence did not support OLP as a risk factor for PIDs. However, this does not mean that implant restoration can be performed indiscriminately in OLP patients. For patients with acute/erosive forms of OLP or desquamative gingivitis, immune system disorder and poor oral hygiene 
may be potential risk factors for PIDs. In clinical settings, whether OLP is well-controlled or not would be a concern during implant treatment planning. The two studies included in the current review had declared that implantation therapy was conducted during the remission stages of OLP. Some study has shown a very high implant failure rate $(42 / 55)$ for OLP patients receiving implant placement during the acute stages [16]. Reversely, controlled OLP patients and healthy condition have displayed an equal marginal bone loss around implant in four-year follow up [38]. Obviously, selecting the remission stages are clinically appropriate and normative. OLP should not be considered as a contraindication for implant treatment in patients who do not have evident symptoms or mucosal erosive congestion and have good oral hygiene. Furthermore, the mental state of OLP patients might be improved after implant treatment, which in turn is better for OLP control, considering that those without implant treatment have been reported to have a poor quality of life, a weaker psychological profile and greater stress [15, 17]. Additionally, for OLP patients receiving glucocorticoid treatment, assessment of alveolar bone mineral density in the area to be implanted should be considered. Finally, it is worth noting the effect of implants in mouth on the recovery of OLP patients, although there were no significant differences in OLP signs and symptoms between the implant group (14 patients) and nonimplant group (15 patients) during the 12-24 month follow-up period [39]. For OLP patients who have undergone implant restoration, OLP conditions may need to be monitored frequently, and implant maintenance planning may need to be personalized.

\section{Implications for further research}

This review systematically analyzed the existing evidence on relation between OLP and PIDs for the first time. Large-scale prospective trials are required to validate the findings.

The results suggest that OLP is not a potential risk factor for PIDs during the 1 to 10-year follow-up period. However, given the relatively small amount of evidence available, the final answer to this question depends on large, well-designed prospective and randomized clinical trials in the future. At present, the proportion of people undergoing implant treatment and the prevalence of OLP worldwide are not high, which may cause difficulty in researching the correlations between these two diseases; therefore, multicenter cooperative clinical research is needed. Additionally, OLP has various clinical classifications, and the prospective study included in this systematic review showed that OLP patients with desquamative gingivitis had a higher frequency of PIM. This suggests that in future studies, subgroup analysis of
OLP may be helpful in exploring the associations between OLP and PIDs. Moreover, interventional studies on the effects of OLP treatment on peri-implant status are still lacking; these studies can further elucidate the associations between OLP and PIDs. Molecular biology techniques can also help to explore the microscopic effects of OLP on peri-implant pathophysiological processes at molecular level.

\section{Conclusions}

Available articles regarding the effects of OLP on PIDs remains very limited. Existing evidence does not support OLP as a suspected risk factor for PIDs. Large-scale prospective trials are required to validate the findings.

\section{Abbreviations \\ OLP: Oral lichen planus; PIDs: Peri-implant diseases; RR: Risk ratio; Cl: Confidence interval; PIM: Peri-implant mucositis; PI: Peri-implantitis; PRISMA : Preferred Reporting Items for Systematic Review and Meta-analyses; NOS: Newcastle-Ottawa Scale; AHRQ: Agency for Healthcare Research and Quality}

\section{Acknowledgements}

The authors thank Liting Yan and Yinshen Yang for their help with the metaanalysis work.

\section{Authors' contributions}

XX and TX formulated the inclusion and exclusion criteria, article search and screening. XX and TY analyzed and interpreted the data and drafted the manuscript. XW, WQ and LG designed and supervised the study and edited and provided final approval for the manuscript to be published. All authors read and approved the final manuscript.

\section{Funding}

This study was supported by the National Natural Science Foundation of China, Beijing, China, Grants 81700985 and 81271159. The role of funding agencies in research: data collection, analysis, and manuscripts.

\section{Availability of data and materials}

The datasets used and/or analyzed during the current study are available from the corresponding author on reasonable request.

Ethics approval and consent to participate Not Applicable.

Consent for publication

Not applicable.

\section{Competing interests}

The authors declare that they have no competing interests.

Received: 26 February 2020 Accepted: 6 May 2020

Published online: 20 May 2020

References

1. Pjetursson BE, Brägger U, Lang NP, Zwahlen M. Comparison of survival and complication rates of tooth-supported fixed dental prostheses (FDPs) and implant-supported FDPs and single crowns (SCs). Clin Oral Implants Res. 2007;18(Suppl 3):97-113.

2. Pjetursson BE, Valente NA, Strasding M, Zwahlen M, Liu S, Sailer I. A systematic review of the survival and complication rates of zirconia-ceramic and metal-ceramic single crowns. Clin Oral Implants Res. 2018;29(Suppl 16): 199-214.

3. Rinke S, Ohl S, Ziebolz D, Lange K, Eickholz P. Prevalence of periimplant disease in partially edentulous patients: a practice-based cross-sectional study. Clin Oral Implants Res. 2011;22(8):826-33. 
4. Heitz-Mayfield LJA, Salvi GE. Peri-implant mucositis. J Periodontol. 2018; 89(Suppl 1):S257-s66.

5. Berglundh T, Armitage G, Araujo MG, Avila-Ortiz G, Blanco J, Camargo PM, Chen S, Cochran D, Derks J, Fiquero E, et al. Peri-implant diseases and conditions: Consensus report of workgroup 4 of the 2017 World workshop on the classification of periodontal and Peri-implant diseases and conditions. J Periodontol. 2018;(89 Suppl 1):S313-s8.

6. Gurgel BCV, Montenegro SCL, Dantas PMC, Pascoal ALB, Lima KC, Calderon PDS. Frequency of peri-implant diseases and associated factors. Clin Oral Implants Res. 2017;28(10):1211-7.

7. BinShabaib MS, Mehmood A, Akram Z, SS AL: Peri-implant clinical and radiographic status and whole salivary cotinine levels among cigarette and waterpipe smokers and never-smokers. J Oral Sci 2018, 60(2):247-252.

8. Schwarz F, Derks J, Monje A, Wang HL. Peri-implantitis. J Periodontol. 2018; 89:S267-S90.

9. Al-Sowygh ZH, Ghani SMA, Sergis K, Vohra F, Akram Z. Peri-implant conditions and levels of advanced glycation end products among patients with different glycemic control. Clin Implant Dent Relat Res. 2018;20(3):345-51.

10. Carrozzo M, Thorpe R. Oral lichen planus: a review. Minerva Stomatol. 2009; 58(10):519-37.

11. Cheng YS, Gould A, Kurago Z, Fantasia J, Muller S. Diagnosis of oral lichen planus: a position paper of the American Academy of Oral and maxillofacial pathology. Oral Surg Oral Med Oral Pathol Oral Radiol. 2016;122(3):332-54.

12. Sugerman PB, Savage NW, Walsh L, Zhao ZZ, Zhou XJ, Khan A, Seymour GJ, Bigby M. The pathogenesis of oral lichen planus. Crit Rev Oral Biol Med. 2002;13(4):350-65.

13. Boorghani M, Gholizadeh N, Taghavi Zenouz A, Vatankhah M, Mehdipour M. Oral lichen planus: clinical features, etiology, treatment and management; a review of literature. J Dent Res Dent Clin Dent Prospects. 2010;4(1):3-9.

14. Rai NP, Kumar P, Mustafa SM, Divakar DD, Kheraif AA, Ramakrishnaiah R, Vellapally S, Dalati MH, Parine NR, Anil S. Relation between periodontal status and pre-cancerous condition (Oral lichen Planus): a pilot study. Adv Clin Exp Med. 2016;25(4):763-6.

15. López-Jornet P, Camacho-Alonso F, Sánchez-Siles M. Dental implants in patients with oral lichen planus: a cross-sectional study. Clin Implant Dent Relat Res. 2014;16(1):107-15.

16. Khamis AK, Aboushelib MN, Helal MH. Clinical management protocol for dental implants inserted in patients with active lichen Planus. Part II 4-year follow-up. J Prosthodont. 2019;28(5):519-25.

17. Pippi R, Patini R, Ghiciuc CM, Sandu RB, Pasquali V, Scaccianoce S, DimaCozma LC, Patacchioli FR. Diurnal trajectories of salivary cortisol, salivary aamylase and psychological profiles in oral lichen planus patients. J Biol Regul Homeost Agents. 2014;28(1):147-56.

18. Petruzzi M, De Benedittis M, Cortelazzi R, Milillo L, Lucchese A, Serpico R, Grassi FR. Implant rehabilitation in patients with oral lichen planus: an overview. Clin Oral Investig. 2012;16(5):1347-52.

19. Anitua E, Piñas L, Escuer-Artero V, Fernández RS, Alkhraisat MH. Short dental implants in patients with oral lichen planus: a long-term follow-up. Br J Oral Maxillofac Surg. 2018;56(3):216-20.

20. Renvert $\mathrm{S}$, Aghazadeh A, Hallström H, Persson GR. Factors related to periimplantitis - a retrospective study. Clin Oral Implants Res. 2014;25(4):522-9.

21. Pesce P, Menini M, Tommasato G, Patini R, Canullo L. Influence of modified titanium abutment surface on peri-implant soft tissue behaviour: a systematic review of histological findings. Int J Oral Implantol (New Malden). 2019;12(4):419-29.

22. Salvi GE, Aglietta M, Eick S, Sculean A, Lang NP, Ramseier CA. Reversibility of experimental peri-implant mucositis compared with experimental gingivitis in humans. Clin Oral Implants Res. 2012;23(2):182-90.

23. Stang A. Critical evaluation of the Newcastle-Ottawa scale for the assessment of the quality of nonrandomized studies in meta-analyses. Eur J Epidemiol. 2010;25(9):603-5.

24. Zeng X, Zhang Y, Kwong JS, Zhang C, Li S, Sun F, Niu Y, Du L. The methodological quality assessment tools for preclinical and clinical studies, systematic review and meta-analysis, and clinical practice guideline: a systematic review. J Evid Based Med. 2015;8(1):2-10.

25. Sterne JA, Sutton AJ, loannidis JP, Terrin N, Jones DR, Lau J, Carpenter J, Rücker G, Harbord RM, Schmid CH, et al. Recommendations for examining and interpreting funnel plot asymmetry in meta-analyses of randomised controlled trials. BMJ. 2011;343:d4002.

26. Fu L. A L, Li CY, Zhou YM: [research progress of dental implant in patients with oral lichen planus]. Zhonghua Kou Qiang Yi Xue Za Zhi. 2019;54(2):135-7.
27. Hernández G, Lopez-Pintor RM, Arriba L, Torres J, de Vicente JC. Implant treatment in patients with oral lichen planus: a prospective-controlled study. Clin Oral Implants Res. 2012;23(6):726-32.

28. Chrcanovic BR, Cruz AF, Trindade R, Gomez RS: Dental Implants in Patients with Oral Lichen Planus: A Systematic Review. Medicina (Kaunas, Lithuania) 2020, 56(2).

29. Chen L, Yang T, Yang G, Zhou N, Dong H, Mou Y. Retrospective clinical analysis of risk factors associated with failed short implants. Clin Implant Dent Relat Res. 2020;22(1):112-8.

30. Simark-Mattsson C, Bergenholtz G, Jontell M, Eklund C, Seymour GJ, Sugerman PB, Savage NW, Dahlgren UI. Distribution of interleukin-2, -4 -10 , tumour necrosis factor-alpha and transforming growth factor-beta mRNAs in oral lichen planus. Arch Oral Biol. 1999;44(6):499-507.

31. Lo Russo L, Guiglia R, Pizzo G, Fierro G, Ciavarella D, Lo Muzio L, Campisi G. Effect of desquamative gingivitis on periodontal status: a pilot study. Oral Dis. 2010;16(1):102-7.

32. Alrashdan MS, Cirillo N, McCullough M. Oral lichen planus: a literature review and update. Arch Dermatol Res. 2016;308(8):539-51.

33. Gualini F, Berglundh T. Immunohistochemical characteristics of inflammatory lesions at implants. J Clin Periodontol. 2003;30(1):14-8.

34. Persson GR, Renvert $\mathrm{S}$. Cluster of bacteria associated with peri-implantitis. Clin Implant Dent Relat Res. 2014;16(6):783-93.

35. Wang K, Lu W, Tu Q, Ge Y, He J, Zhou Y, Gou Y, Van Nostrand JD, Qin Y, Li $\mathrm{J}$, et al. Preliminary analysis of salivary microbiome and their potential roles in oral lichen planus. Sci Rep. 2016;6:22943.

36. González-Moles MA, Scully C. Vesiculo-erosive oral mucosal disease-management with topical corticosteroids: (2) protocols, monitoring of effects and adverse reactions, and the future. J Dent Res. 2005;84(4):302-8.

37. Verhoeven AC, Boers M. Limited bone loss due to corticosteroids; a systematic review of prospective studies in rheumatoid arthritis and other diseases. J Rheumatol. 1997;24(8):1495-503.

38. Aboushelib MN, Elsafi MH. Clinical management protocol for dental implants inserted in patients with active lichen Planus. J Prosthodont. 2017; 26(1):29-33.

39. Czerninski R, Eliezer M, Wilensky A, Soskolne A. Oral lichen planus and dental implants--a retrospective study. Clin Implant Dent Relat Res. 2013; 15(2):234-42.

\section{Publisher's Note}

Springer Nature remains neutral with regard to jurisdictional claims in published maps and institutional affiliations.

Ready to submit your research? Choose BMC and benefit from:

- fast, convenient online submission

- thorough peer review by experienced researchers in your field

- rapid publication on acceptance

- support for research data, including large and complex data types

- gold Open Access which fosters wider collaboration and increased citations

- maximum visibility for your research: over $100 \mathrm{M}$ website views per year

At BMC, research is always in progress.

Learn more biomedcentral.com/submissions 